Produkten nach Patent 252705, dadurch gekennzeichnet, daß an Sielle der oxydierten Oele Gemische von oxydierten Oelen und Phenolen verwendet werden, wobei die Menge der Phenole die des oxydierten Oeles nicht übersteigen darf. Zweitens Ausiuhrungsform des Verfahrens nach Anspuch 1, gekennzeichnet durch den Zusatz von festen, indifferenten Stoffen oder Füllmitteln zum Reaktionsgemisch.

\section{Neue Patente.}

\section{Anmeldungen.}

12d. F. 33299 . Verfahren und Vorrichtung zum Filtrioren and Trocknen von Kollolden. Dr. Walter Fienming, BerlinSchaneberg, Heilbronnerstrabe 4. 1.11.11.

29 b. H 55655 . Verfahren zar Herstellung künstlicher SeidenIiden aus in Kupferoxydammoniak peiöster Zellulose unler Verwendung von Aetzalkalilauge als Fultmiltel. Hanauer Kunstseidefabrik Akt-Ges, Grof-Auheim bei Hanau a. 13. 10. 11 .

29b. S. 34983 . Verfahren zur Herstellung von kiinstlichem RoBhaar. Claude Marie Saniaville, Le Coteau, Frankr.: Vertr.: A.elliot u. Dr.A.Manasse, Pat.-Anwalte, Berlin $\mathrm{SW.48}$ 911.11

30h. L. 32499 . Verfahren zur Herstellung zelluloidähnlicber Massen. Feodor Lehmann, Claudiusetr. 5, thd Johannes Stocker Korsörerstr. 12, therlin. 1. 6 .11.

39b. R. 32895. Vetfahren zur Her-tellung eines Gummiersatzes aus Oelen und Chiorschwefel unter Mitworkung geeigneter Harze und Neutralisationsmiltel. Rubber Substitute (1910) Limited, London; Vertr.: Lipl.-Ing. E. Dippel, Pat.-Anw. Frankfurt 2. M. 3 4. it.

12d. F. 33300. Verfahren und Vorrichtung zum Filtrieren und Trocknen vou Kolloiden und zum Reinigen der berm Filtrieren benutzten Filterflächen: Zus. Z. Antm. F. 33299 . Dr.Waluer Flemming, Berlin-Schöneberg, Heilbronnessir. 4. 1. 11. 11 .

12i. S. 35989 . Verfahren zur Herstelltung von Kteselsäture aus Alkalistlikatlöøungen und Kohlensäure. Johann Leonhard Seyboth, Munchen, Ysenburgstr. 11. 27, 3, 12,

22e. B 65587 . Vesfahren zur Darsielinty von indigolden Farbstoften. Badische Anilin- \& Soda-Fabrik, Ludwigshafen a. Rh. 18.1211 .

22 h. B. 60376 . Verfabren zur Darstellung von Lacken aus Zellutoseestern: Zus. z. Pat. 251 351. Badische Anilin\& Soda-Fabrik, Ludwigshafen a. Rh. 23. 2. 12

20b. H. 56146. Verfaluren zur Herstellung von künstlichen Seldenfäden aus Kupteroxydammoniakzeliuloselösung tach dem Streckspinnverfahren. Hanater Kunstsejdetabrik A. 0 Groß-Auteim b. Hanau a. M. 2.12.11.

39b. B. 61984 Verfahren zur Herstellung einer bernsteineGhllchen Masse. J. Bahret, Stuttgart, Kötigshau. $15,2.11$.

39 b. D 26854 . Verfahren zur Reinigung von Kautschuk Zus.z. Pat. 244712. Henry Pierre Charles Georges Debauge, paris; Vertr.: C. Gronert u. $W$. Zimmermann, Pat.-Anwalte, Berlin SW.61. 19.4.12.

39b. P, 28261. Verfahren zum Kongulieren des Millchsaftes von Kautschuk liefernden Pflanzen; Zus. Z. Pat. 237789. Fa. Wilhelm Pahl, Dortuntur, 2 2.12.

85b. K. 51155 . Verfatiren und Vorrichtung zur Reinlgung nat wrlicher wasser mittelx Bestrahlung durch Sonwenoder Tageslicht. August Kock, Hannover-List. $23,4,12$.

2g. W 37761 . Verfahren zur Herbeifuhrung chemischer Roaktionen awlschen Flügsigkelten und Gasen unter Verwendung von katalyti.ch wirksamen Stoffon, oder chemisch wirksamen Strahlen. Dr. Johann Walter, Genf Vertr.: Albert Rheir, Weil Nr. 27, Amt Lörach i. Bad. 26.71 .

\section{Erteilungen.}

57b. 252337. Verfahren zur Schwefeltosung von Silberbildern. Chemische Werke vorm. Dr. Heinrich Byk, Chariottenburg. 13. 1211 . C 21366.

12d. 252640 . Verfahren zur Gewinnung und Wiederbelebune von Euttürbuteskohle. Oskar Molenda u. Josef Wunsch, Skrivan b Neubidschow; Vertr.: Dr. J. Ephraim; Pat.-Anw., Berlin SW $11,134.12$. M. 47531

120. 252706 . Verfahren zur Hersteling hydratisierter Zelloloseester. Farbentabrikert vorm. Friedr. Bayer \& Co., Elberfeld. 30.905. M. 28289 .

41. 254496. Verfahren zur Herstellang von Kunsteeldeglöhkörpern unter fallung des Thors des mit verbindungen der Leuchterden getränkien Strumpies als ThorhydroxydDr. Fritz Wirth, Berlin -Wilmersdorf, Landauerstr. 1, 21.7.11. W. 37717 .

39.b. 254385. Verfahren zur Hersiellung von Lösungen aus axetonlosillcher Axetylzelłulose. "Dr, Arthur Eichengrün, Berin, Konstanzerstr. 87, 26, 1,09. E. 14310

85c. 254294 . Verfahren zur Behandlung des Abwasserschlamms in Klaranlagen. Julkus Neumann: Berlin, Tile Wardenbergstrabe $6.17,969, \mathrm{~N}, 10942$

29k. 252661 . Verfahren zur Hersiellung von Zelluloselösunzeg: Zus. z. Fat. 245 575. Dr. Wilhein Traube, Berlin, Motzstr. 90 . 6.8 $11 . T .16908$

120. 252759. Verfahren zut elektrolytischen Behandlung organlscher Körper. Farbenfabriken vorm. Friedr. Bayer \& Co. Elber feld, 25.6.11. F. 32613

12k. 254437. Verfahren zur Hersteilung von Ammosiak aus semen Elrmenten mit Hilfe von Kaialysatoren: Zus. z. Pat. 249447. Badische Anilin- Bo Soda-Fabtik, Ludwighafen a. Rh. 22. 4. 10. B. 58377 .

120. 254 474. Verfahren zur Horstellung von Harsstoft aus Cyananid unter Verwendung eines katalysators. Dr. Heinrich Iminendorft, Westendstr, 13, w, Dr. Hubert Kappen, Reichardstieg 14, Jenz. 20. 9. 10. I. 12964.

12p. 254480 Verintren Zut Darstellung elwetishaltiger leicht resorbietbarer Bisensalze der in den Pflanzen enthaltenten ressimilierbaran Phosphorverbindung (Inositphosphorsäure). Gesellschafi für Chemische Industrie in Basel, Basel; Vertr.: A. Loll, Pat.-Anw., Berlin SW. 43.2 . 7.11. O. 34641 .

$12 \mathrm{q} 254411$ Verfahren zur Hersiellung von harzartigen töslichen Kondensationsprodukten aus Phtenoen und Formaldehyd. Dr. Kurt Albert w. Dr. Ludwig Berend, Amontneburg b. Biebrich a. Rh. 3011,10, A. 19781 .

23e. 254469 Verfahren zur Herstellung von festen, neutraten Seifen mit hohem Gehult an Kohlenwasserstoffen o dgl Ernst Eruno Wolf u. Curt Böhme, Chemnitz, Moritzstr, $29.41,11,09, W, 33316$

\section{Notizen.}

Priv.-Dozent Professor Dr. Woltgang Pauli in Wien wurde zum wirklichen auBerordentlichen Professor an der Wjener Universität ernannt. Derselbe wird auch weiterhin iber Kolloidchemic und physikalisch-chemische Biologie Vorlesungen halten und die physikalisch-chemische Abteilung der biologischen Versuchsanstalt in Wien leiten.

Priv.-Dozent Dr. G. Wi egner wurde zum etatmabigen Professor fur Chemie, spez. Agrikulturchemie, an der forstwissenschaftlichen Abteilung der Techn. Hochschule in Zürich berufen.

Ore nächsten Hefte dier Kolloid-Zeitschrift resp. der Kolloidchemischen Beithefte werden 4 a. folgende Arbeiten enthalten:

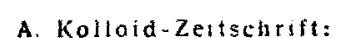

A. Kolloid-Zetschrift:

W. Döhle u. B. Rassow: Ein gelatinöses Quecksilbersalz einer organischen Sulfosẫure.

D. Spence: Einige Remerkungen zur Theorie der Vulkanisation. S. Stafford Whitby: Ueber einige vorläfige Beobachtungen bezüglich der Ursachen natürificher Veränderungen in Latex von Hevea Brasiliensis, und ther die Depolimerisation von Kautschuk und seine Umwandiung.

f. G. Fol: Viskositä́sbestimmung von Kautschuklôsungen.

A. von Rossem: Ueber dis Leimigxerden des Kautschuks.

R. E. Liesegang: Uebet schaliz-disperse Systene.

H. Siedentopf: Hiifsobjektiv für Voruntersichungen zum Kardioid Uitramikroskop.

H. Chlck und C. Martir: Die Dichte and das Lösangsvolamen einiget Proteine.

E. Paterno und F. Medigreceanu: Studien ather kolloide Liosungen.

B. Kolloidebemische Bellofte:

J. Frank: Unterstichungen uther cintige physikalische Eigenschaften kolicider lösumen.

C. Benedicks: Die Natur der cektrischen Kolloidsynthese.

J Neubert: Die Tonverflüssigung durch Alkall.

P. Pon We im arn und Mitarbeiter: Dispersoldchemische Mitteilungen aus dem Laboratoriun für physikalische Chemle des Berginstitutes St. Peter burg usw. s, vor. Hefle der K.-Z, 